\title{
Photophysics and aggregation effects of a triphenylamine-based dye sensitizer on metal-oxide nanoparticles suspended in an ion $\operatorname{trap}^{\dagger}$
}

\author{
Viktoras Dryza, ${ }^{a *}$ Jessica L. Nguyen, ${ }^{a}$ Tae-Hyuk Kwon, ${ }^{b \ddagger}$ Wallace W.H. Wong, ${ }^{b}$ Andrew B. Holmes ${ }^{b}$ \\ and Evan J. Bieske ${ }^{a}$
}

\author{
Received Xth $X X X X X X X X X X 20 X X$, Accepted Xth $X X X X X X X X X 20 X X$ \\ First published on the web $X$ th $X X X X X X X X X X 200 X$ \\ DOI: 10.1039/b000000x
}

The photophysical behaviour of a triphenylamine-based organic dye sensitizer (Carbz-PAHTDTT) attached to alumina and titania nanoparticles (labelled Carbz-Al and Carbz-Ti, respectively) is examined in the absence and presence of the chenodeoxycholic acid (CDCA) coadsorber. The experiments are conducted in vacuo by suspending the target dye-sensitized nanoparticles within a quadrupole ion trap, where they are probed with laser radiation to obtain emission spectra and time-resolved excited state decay curves. For Carbz-Al, the dye's emission band is blue-shifted and the excited state lifetime is increased upon the coabsorption of CDCA, effects attributed to reduced dye aggregation. Compared to Carbz-Al, the Carbz-Ti excited state lifetimes are significantly shorter due to excited dye molecules injecting electrons into the titania conduction band. For Carbz-Ti, the electron injection quantum yields for the surfaces with CDCA (CDCA:dye $=25: 1$ ) and without CDCA are estimated to be 0.87 and 0.71, respectively. The gas-phase results demonstrate that Carbz-PAHTDTT dye aggregates are detrimental to the performance of a dye-sensitized solar cell.

\section{Introduction}

Dye-sensitized solar cells (DSSCs) are promising devices for the efficient and economical conversion of sunlight to electrical energy. ${ }^{1,2}$ Within a DSSC, dye sensitizer molecules anchored to a mesoporous, metal-oxide, anode electrode absorb photons and inject the promoted electrons into the metal-oxide conduction band.

Recent research has demonstrated significant advantages in using organic dyes as the sensitizer, rather than traditional ruthenium polypyridyl coordination complexes; they are cheaper to synthesize, have higher absorption coefficients, and can be electronically tuned to match new types of redox couples. ${ }^{3-5}$ However, unlike ruthenium dyes, organic dyes do not form a long-lived triplet state after photoexcitation, so that the competition between electron injection and ground state recombination becomes a crucial factor in determining a DSSC's overall performance. Dye-sensitized metal-oxide nanoparticles (NPs) and films serve as useful model systems for the DSSC anode and the kinetic events occurring at the dye/surface interface can be explored using time-resolved fluorescence spectroscopy. ${ }^{6-10}$

${ }^{a}$ School of Chemistry, The University of Melbourne, Victoria 3010, Australia.

*E-mail:vdryza@unimelb.edu.au

${ }^{b}$ School of Chemistry, Bio21 Institute, The University of Melbourne, Victoria 3010, Australia.

\$ Present address: Interdisciplinary School of Green Energy, Ulsan National Institute of Science Technology, Ulsan 689-798, Republic of Korea.
Recently, Daeneke et al. synthesized an organic dye sensitizer, labelled Carbz-PAHTDTT, consisting of a triphenylamine donor motif (supplemented with carbazole units), a dithienothiophene bridge motif, and a cyanoacrylic acid acceptor motif (Fig. 1). ${ }^{4}$ Dyes that exploit similar organic subunit combinations are currently amongst the most successful sensitizers for DSSCs. ${ }^{3}$ Desirable features of CarbzPAHTDTT include its strong absorption across most of the visible spectrum and compatibility of its redox properties with ferrocene/ferrocenium-based redox couples, which are being investigated as non-corrosive alternatives to the popular iodide/triiodide redox couple. Interestingly, the highest solar conversion efficiency for the Carbz-PAHTDTT-based DSSC $(\eta=7.5 \%)$ was obtained with the chenodeoxycholic acid (CDCA) coadsorber (Fig. 1) also attached to the titania anode electrode. ${ }^{4}$ The beneficial effects of CDCA have been linked to a reduction in the formation of dye aggregates, $, 7,10-12$ an upward shift in the metal-oxide conduction band, ${ }^{11,13}$ and better shielding of the surface against recombination processes. ${ }^{11,14}$

To gain a clearer picture of a dye sensitizer's intrinsic photophysical properties, it is useful to study the dynamics of dyebased systems in a vacuum, eliminating perturbations induced by solvent or atmospheric environments. ${ }^{15,16}$ In the current study, we demonstrate that in vacuo spectroscopic data can be obtained for dye-sensitized NPs by suspending them in a quadrupole ion trap (QIT) and probing them with laser-based fluorescence techniques. Fluorescence studies of trapped ions

This journal is @ The Royal Society of Chemistry [year]

Journal Name, 2010, [vol], $\quad 1-8 \mid 1$ 


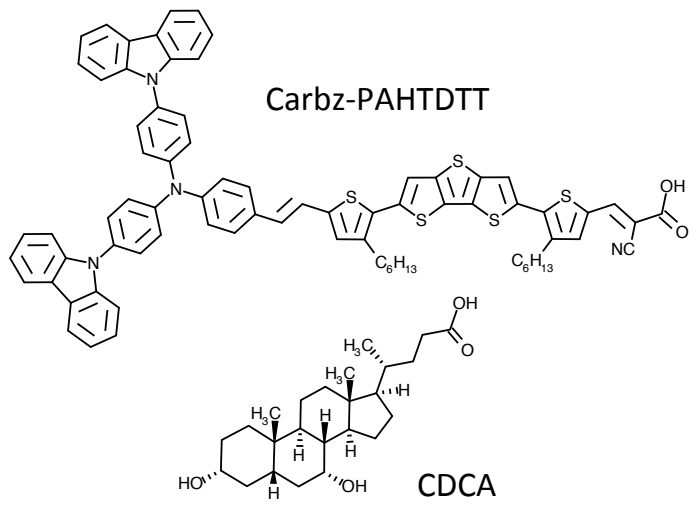

Fig. 1 Structures of the Carbz-PAHTDTT dye sensitizer and chenodeoxycholic acid (CDCA) coabsorber

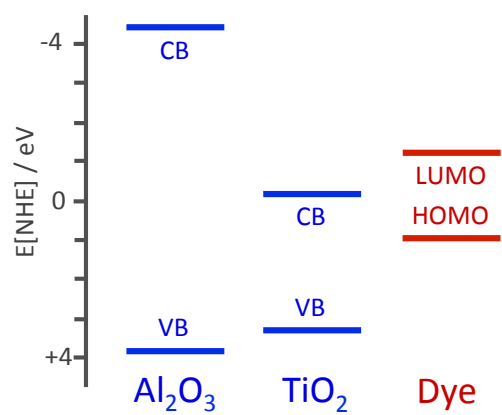

Fig. 2 Band edge positions for the alumina and titania metal-oxide surfaces and the Carbz-PAHTDTT dye sensitizer's molecular orbital positions

are challenging due to low ion densities. Nevertheless, this approach has been successfully applied to molecular ions and nano- to micron-sized particles suspended within QITs. ${ }^{17-24}$

Here, we utilize laser-induced acoustic desorption (LIAD) to introduce charged Carbz-PAHTDTT-sensitized metal-oxide NPs (Carbz-NPs) into a purpose-built QIT and characterize their photophysical properties using emission spectroscopy and time-resolved fluorescence measurements. Titania $\left(\mathrm{TiO}_{2}\right)$ and alumina $\left(\mathrm{Al}_{2} \mathrm{O}_{3}\right)$ NPs are used because DSSCs are commonly fabricated with a titania anode electrode, whereas alumina serves as an inert reference substrate. Band edge positions for the metal-oxide surfaces and the molecular orbital levels for the Carbz-PAHTDTT dye are illustrated in Fig. 2; the low conduction band edge of titania allows electron injection, whereas the high conduction band edge of alumina prohibits electron injection. ${ }^{4,25}$ Ultimately, the CarbzPAHTDTT lifetimes for excited state relaxation and electron injection, measured in the absence and presence of CDCA, allow the effect of dye aggregates on the light-harvesting efficiency to be evaluated.

\section{Experimental approach}

The Carbz-NPs were prepared as follows. A $0.2 \mathrm{mM}$ solution of the Carbz-PAHTDTT dye (synthesis given in Ref. 4) and a $2 \mathrm{mM}$ solution of CDCA (Sigma-Aldrich), both in chloroform/ethanol (60/40, v/v), were used to make sensitizing solutions with CDCA:dye ratios of $0: 1,1: 1,5: 1$, and $25: 1$. To $3 \mathrm{~mL}$ of the sensitizing solution, $4 \mathrm{mg}$ of NPs was added (NanoAmor: $\mathrm{Al}_{2} \mathrm{O}_{3}$ 20-30 nm gamma, Sigma Aldrich: $\mathrm{TiO}_{2}<25 \mathrm{~nm}$ anatase) and sonicated for 60 minutes. The amount of dye/CDCA was sufficient to obtain monolayer coverage of the NPs; dyes with cyanoacrylic acid anchoring groups generally have $\sim 2$ molecules $/ \mathrm{nm}^{2} .{ }^{26}$ The solutions were then centrifuged, with the excess solution removed, followed by the addition of $1 \mathrm{~mL}$ chloroform/ethanol solution and 10 minutes sonication. This was repeated a further two times to remove any dye not bound to NP surface.

LIAD has proven to be a versatile matrix-free method for the soft desorption of microparticles and biological cells into a vacuum environment. ${ }^{23,27}$ For the LIAD technique, a few drops of the Carbz-NP solution was placed on the front surface of a $0.3 \mathrm{~mm}$ thick silicon wafer and then air dried. The wafer was then placed into the vacuum chamber and held just above the QIT. The rear of the wafer was irradiated with a single, focussed laser pulse $(532 \mathrm{~nm}, \sim 7 \mathrm{~ns}, 40-60 \mathrm{~mJ})$ from a Q-switched Nd:YAG (Continuum, Surelight), causing an acoustic shock that propeled several hundred charged particles from the front wafer surface into the QIT. Nitrogen buffer gas (40 mTorr) present in the QIT helped dissipate the initial kinetic energy of the desorbed Carbz-NPs, enhancing the trapping efficiency, and thereafter collisionally cooled the CarbzNPs heated by the excitation laser beam.

The QIT used in this study (Fig. 3) is based on the design of Schlemmer et $a l^{28}$ and has previously been used to investigate single micron-sized particles. ${ }^{22,23}$ The QIT consists of 2 conical end-cap electrodes surrounded by 8 thin parallel rods (which together act as the ring electrode). This open arrangement allows the QIT to be easily loaded by LIAD and provides facile access for excitation laser beams and fluorescence collection. The QIT was operated in single-phase mode, whereby an oscillating potential ( $f=1 \mathrm{kHz}$, amplitude $=200 \mathrm{~V}$ ) was applied to the end-cap electrodes, while the parallel rods were grounded. The polarity of the ion population was determined by observing its movement when a potential was applied between the top two and bottom two parallel QIT rods.

When the trapped ion cloud was illuminated by the laser beam, clearly visible light was elastically scattered from a cylindrical region of $\sim 1 \times 4 \mathrm{~mm}(\varnothing \times h)$ within the QIT, indicating that the majority of the Carbz-NPs were present as submicron-sized clusters, rather than isolated NPs. Indeed, the QIT conditions corresponded to ions being trapped that had 1 charge for every $\sim 2-10$ Carbz-NPs, highlighting the 


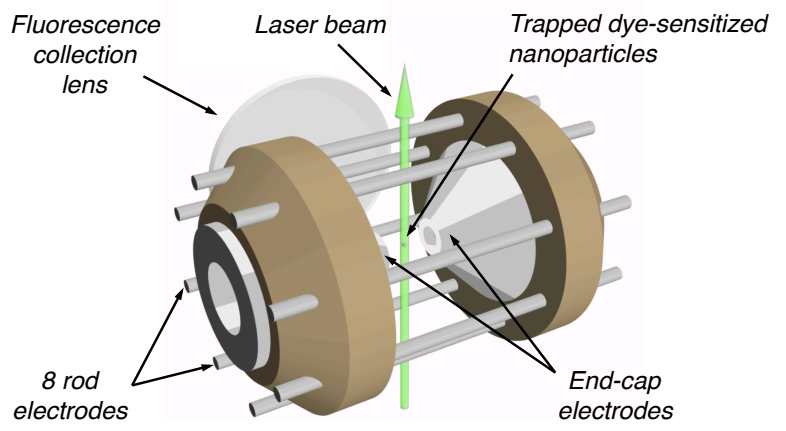

Fig. 3 Quadrupole ion trap (QIT) used to spectroscopically investigate Carbz-NPs in the gas phase

ability of LIAD to generate low charge species. The trapped ion cloud usually consisted of negative ions, but occasionally was composed of positive ions. The spectroscopic data (emission spectra, fluorescence lifetimes) were independent of trapping frequency $(0.5-2.0 \mathrm{kHz})$ and population polarity (+ or -), implying that the effect of charge is negligible for Carbz-NP clusters in this mass-to-charge range.

For the dispersed emission experiments, trapped CarbzNPs were probed with loosely focussed $532 \mathrm{~nm}$ radiation $\left(\sim 500 \mathrm{~mW} / \mathrm{cm}^{2}\right)$ from a continuous-wave $\mathrm{Nd}: \mathrm{YVO}_{4}$ laser (Coherent, VERDI-V5). Fluorescence from the Carbz-NPs was collected by a F/1 lens, passed through a $532 \mathrm{~nm}$ long pass filter, and sent to a spectrometer (Jobin Yvon, Triax 552) interfaced with a cooled 2-dimensional charge-coupled device detector (Jobin Yvon, Symphony). For the time-correlated single photon counting (TCSPC) experiments, trapped Carbz-NPs were probed with loosely focussed, pulsed $532 \mathrm{~nm}$ radiation $\left(\sim 12 \mathrm{ps}, \sim 500 \mathrm{~mW} / \mathrm{cm}^{2}, 82 \mathrm{MHz}\right)$ from a picosecond, diodepumped Nd:YAG laser (Time Bandwidth Products, Cheetah$\mathrm{X})$. Fluorescence was collected by a F/1 lens, passed through a $532 \mathrm{~nm}$ long pass filter, and sent to a cooled photomultiplier tube (Becker and Hickl, PMC-100) connected to a TCSPC card (Becker and Hickl, SPC-630). To record the instrument response function (IRF), the $532 \mathrm{~nm}$ long pass filter was removed and the elastically scattered light was monitored. Emission spectra were recorded before and after the TCSPC experiments to ensure the sample had not degraded. The timeresolved fluorescence decay curves were fitted to a stretched exponential function convoluted with the IRF (FAST software, Edinburgh Photonics). Errors in the average lifetimes were estimated to be $\pm 0.02 \mathrm{~ns}$ for the Carbz-Al decays and $\pm 0.04 \mathrm{~ns}$ for the Carbz-Ti decays. A biexponential function was also trialled to fit the data, but was found to provide poorer fits for the accurately recorded Carbz-Al data, compared to the stretched exponential function.

\section{Results and discussion}

\subsection{Carbz-NP photophysics}

Before examining the spectroscopic data, it is useful to consider the important photophysical processes that occur when an organic dye sensitizer assembles onto a metal-oxide surface. Because organic dyes have relatively planar structures, two or more dyes can bind together through reasonably strong intermolecular $\pi-\pi$ interactions to form dye aggregates. ${ }^{7}$ The dye aggregate's properties (e.g., excitation energy, excited state decay rates) can be quite different to those of the dye monomer. ${ }^{29}$ Dye aggregates are usually defined as being either J- or H-aggregates, depending on whether their absorption band is red- or blue-shifted, respectively, from that of the monomer. CDCA has been shown to be an effective antiaggregation agent when coabsorbed with the dye onto a metaloxide surface. ${ }^{7,8,10-12}$ The saturated structure of CDCA means that it has weak intermolecular interactions with the dye and does not absorb visible light.

The photoexcited dye molecule/aggregate can decay through either radiative or non-radiative means. Fluorescence from the excited state to the ground state is usually the slowest relaxation mechanism. Possible non-radiative relaxation mechanisms include internal conversion to the ground state, intermolecular resonance energy transfer to an adjacent dye molecule/aggregate, or injecting an electron into the metaloxide conduction band. Electron injection requires that the conduction band is energetically accessible from the dye's $\pi^{*}$ lowest unoccupied molecular orbital and that there is adequate electronic coupling between the two. Intersystem crossing from the excited singlet state into the triplet state is considered to be negligible, as this process is slow $(\sim n s)$ in organic dyes compared to the other pathways $(\sim \mathrm{ps}){ }^{7}$

For the dye-coated NPs investigated in this work, the emission spectra of the dye monomer and dye aggregates overlap, rendering it impossible to obtain their individual fluorescence decay curves when a mixture is present. Therefore, the measured excited state decays represent the average behaviour of the dye monomer and aggregate mixture, with the observed decay rate $\left(k_{o b s}\right)$ for each sample representing a sum of phenomenological rate constants for radiative relaxation $\left(k_{R}\right)$, internal conversion $\left(k_{i c}\right)$, and electron injection $\left(k_{i n j}\right)$ :

$$
k_{o b s}=k_{R}+k_{i c}+k_{i n j}
$$

\subsection{Emission spectra}

For the gas-phase spectroscopic experiments, the suspended Carbz-NPs are excited with $532 \mathrm{~nm}$ laser light. As can be seen from the absorption spectrum of Carbz-PAHTDTT in solution (Fig. 4), light at this wavelength excites the $S_{1} \leftarrow S_{0}$ electronic transition, which has an absorption maximum 


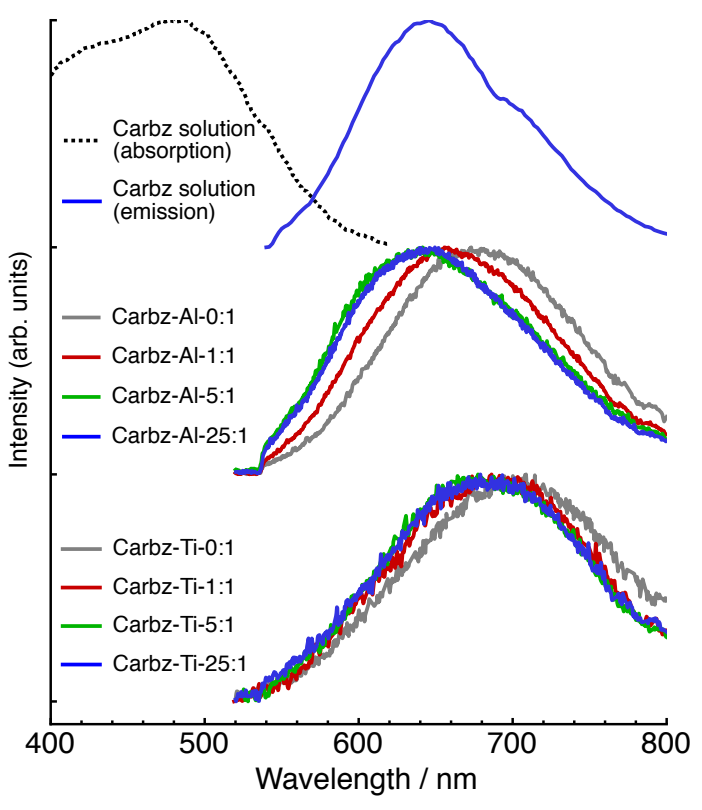

Fig. 4 Normalized absorption and emission spectra of the Carbz-PAHTDTT dye sensitizer in dichloromethane (top), normalized emission spectra of the Carbz-Al samples in the gas phase (middle), and normalized emission spectra of the Carbz-Ti samples in the gas phase (bottom)

at $490 \mathrm{~nm}$, and is predicted to have $\pi$ - $\pi^{*}$ intramolecular charge-transfer character. ${ }^{4,30,31}$ Excitation of the solutionphase Carbz-PAHTDTT at $532 \mathrm{~nm}$ yields a broad emission band with a maximum at $645 \mathrm{~nm}$ (Fig. 4).

Gas-phase emission spectra recorded for alumina CarbzNPs (Carbz-Al) and titania Carbz-NPs (Carbz-Ti) are also shown in Fig. 4, with emission maxima listed in Table 1. Experiments are conducted using Carbz-NPs prepared from sensitizing solutions with CDCA:dye ratios of 0:1 (i.e., only dye), $1: 1,5: 1$, and 25:1. Because the number of ions injected into the QIT with each LIAD shot varies and there is no way to determine the number of trapped ions, the intensity of each emission spectrum is normalized. However, the fluorescence intensity is considerably weaker for Carbz-Ti compared to CarbzAl. The gas-phase emission spectra for all Carbz-NP samples are similar in appearance, displaying a broad band with a single maximum, close to the solution-phase maximum.

For Carbz-Al-0:1, the emission band has a maximum at $680 \mathrm{~nm}$, whereas the presence of CDCA in Carbz-Al-1:1 causes the maximum to blue-shift to $660 \mathrm{~nm}$. The emission bands of Carbz-Al-5:1 and Carbz-Al-25:1 are essentially the same and display a further blue-shift of the maximum to $645 \mathrm{~nm}$. The progressive blue-shift of the maximum is consistent with CDCA molecules becoming interleaved between the dye molecules, gradually breaking up the dye aggregates and eventually leaving only dye monomers on the surface. The blue-shift in the emission arises because the emission wavelength increases with dye aggregate size. Incrementally increasing the CDCA concentration in the sensitizing solutions eliminates first the formation of larger dye aggregates (e.g., trimers), followed by smaller dye aggregates (e.g., dimers). That multiple emission maxima are not evident (i.e., one for each dye aggregate size) and that the widths of the emission bands are approximately the same suggest that intermolecular resonance energy transfer occurs on the surface, whereby the dye monomers and aggregates funnel their electronic energy to the longest wavelength absorbing species. An alternate explanation is that there is only a narrow dye aggregate size distribution in each sample, although this is unlikely as previous studies have found that even without CDCA the majority of the dye molecules exist as monomers. ${ }^{11,32,33}$ Despite similar emission trends and mechanisms being proposed in previous studies, ${ }^{8,34}$ a detailed understanding of how CDCA reduces dye aggregation is still being developed. Recent computational work suggests that intermolecular dyeCDCA complexes within the sensitizing solution may be important precursors in determining the surface-bound structures formed. ${ }^{35,36}$

As the emission spectra of Carbz-Al-5:1 and Carbz-Al-25:1 are essentially the same, it appears that the majority of dye aggregates are eliminated once the CDCA:dye ratio exceeds 5:1. Therefore, the Carbz-Al-25:1 emission spectrum should represent that of the isolated dye monomer.

The Carbz-Ti-0:1, Carbz-Ti-1:1, Carbz-Ti-5:1, and CarbzTi-25:1 gas-phase emission spectra display maxima at 705, 690, 685, and $685 \mathrm{~nm}$, respectively. As for Carbz-Al NPs, the emission bands are blue-shifted with increasing CDCA concentration, with the Carbz-Ti-5:1 and Carbz-Ti-25:1 bands being essentially identical due to elimination of dye aggregates. However, there are several notable differences between the Carbz-Al and Carbz-Ti spectra. First, the magnitude of the blue-shift accompanying addition of CDCA is reduced for the Carbz-Ti NPs. This is believed to be due to rapid electron injection into the titania conduction band, which quickly deactivates the excited dye monomers, reducing the quantum yield for intermolecular resonance energy transfer to the dye aggregates, which emit at longer wavelengths. Therefore, the emission mainly originates from the dye monomer/aggregate population initially excited by the laser, rather than from dye aggregates energized through resonance energy transfer. Second, the emission maximum from the dye monomer on titania (i.e., Carbz-Ti-25:1) is slightly broader and $\sim 0.1 \mathrm{eV}$ lower in energy than on alumina (i.e., Carbz-Al-25:1). This effect is attributed to a strong bond being formed between the dye's carboxylate anchor and the titania surface; compared to the $\mathrm{p}$ orbitals of aluminium, the $\mathrm{d}_{\pi}\left(\mathrm{t}_{2 g}\right)$ orbitals of titanium have better spatial arrangement for electronically coupling with the dye's $\pi^{*}$ states. 
It is not possible to determine the nature of the CarbzPAHTDTT dye aggregates without measuring the Carbz-NPs' absorption spectra. A red-shift in the emission band upon aggregation could arise from either J-aggregates that have roughly equal or larger Stokes shifts than the monomer, or $\mathrm{H}$-aggregates that have much larger Stokes shifts than the monomer. However, for efficient intermolecular resonance energy transfer, the emission band of the monomer should have good spectral overlap with the absorption bands of the dye aggregates, a situation more suited to J-aggregates.

\subsection{Excited state decays}

The time-resolved fluorescence decay curves recorded for the gas-phase Carbz-Al samples are shown in Fig. 5, together with the IRF (half-width $\sim 0.25 \mathrm{~ns}$ ). A notable feature for dye sensitizers attached to metal-oxide surfaces is that the complex nature of the dye/surface interface (e.g., surface inhomogeneities, different dye binding modes, dye aggregates) results in non-exponential fluorescence decays, due to an underlying distribution of numerous first-order processes. ${ }^{6}$ To analyze the Carbz-Al decay curves, a stretched exponential function is employed:

$$
I(t)=I_{0} e^{-\left(\frac{t}{\tau}\right)^{\beta}}
$$

Here, $I_{0}$ is the intensity at time $t=0, \tau$ is the characteristic lifetime, and $\beta$ is the dispersion parameter. The stretched exponential function has been successfully used to model the kinetics of dye-sensitized surfaces probed by time-resolved fluorescence. ${ }^{6,9,10}$ It has the advantages that it minimizes the number of variable parameters used to fit the decays and is compatible with the macroscopic premise of a distribution of first-order lifetimes, with $\beta$ giving a measure of the distribution's breadth (i.e., $\beta=1$ gives a single exponential function typical of a homogeneous system, whereas $\beta<1$ leads to a kinetic distribution). The fitted parameters are used to determine the average lifetime, $\langle\tau\rangle$ :

$$
\begin{aligned}
<\tau> & =\frac{\tau}{\beta} \Gamma\left(\frac{1}{\beta}\right) \\
\Gamma\left(\frac{1}{\beta}\right) & =\int_{0}^{\infty} x^{\frac{1}{\beta}-1} e^{-x} \mathrm{~d} x
\end{aligned}
$$

The fitted and derived parameters for the Carbz-Al samples are given in Table 1, with the fits (convoluted with the IRF) presented in Fig. 5. Generally, the Carbz-Al decays become slower with increasing CDCA concentration. Whereas CarbzAl-0:1 has a lifetime of 0.14 ns, Carbz-Al-1:1 and Carbz-Al5:1 display substantial increases in their lifetimes to 0.33 and $0.41 \mathrm{~ns}$, respectively. Further lifetime increases with higher CDCA concentrations are only minor, with Carbz-Al-25:1 having a lifetime of $0.45 \mathrm{~ns}$. Notably, the $\beta$ parameter also

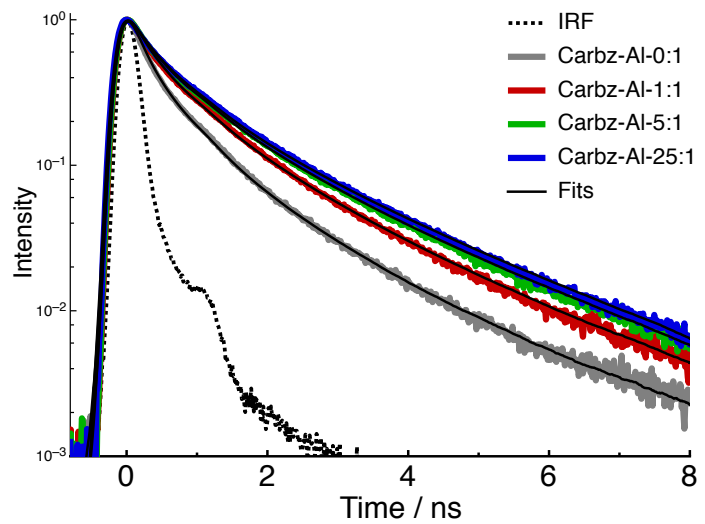

Fig. 5 Time-resolved fluorescence decay curves for the Carbz-Al samples recorded in the gas phase

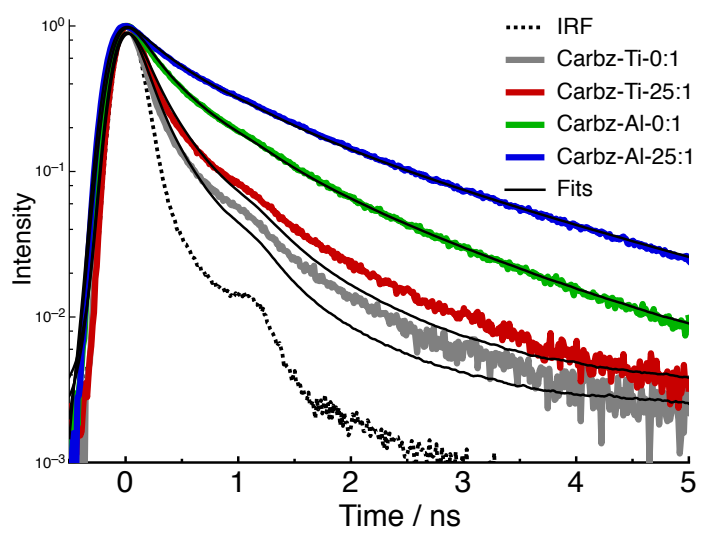

Fig. 6 Time-resolved fluorescence decay curves for the Carbz-Al-0:1, Carbz-Al-25:1, Carbz-Ti-0:1, and Carbz-Ti-25:1 samples recorded in the gas phase

increases moderately with CDCA concentration, possibly due to an increase in the relative number of dye monomers.

The increase in the fluorescence lifetime with CDCA concentration is presumably associated with a reduction in the number of dye aggregates on the surface, with the slow decay of Carbz-Al-25:1 representing the dye monomer's relaxation from the excited state to the ground state. On the other hand, we propose that for Carbz-Al-0:1 both dye monomers and aggregates are initially excited, followed by the excited monomers undergoing intermolecular resonance energy transfer to further increase the excited dye aggregate population. The dye aggregates relax to the ground state more rapidly than the monomer due to superradiance effects arising from coherent coupling of the interacting monomers' transition dipole moments. ${ }^{37-40}$ An enhanced excited state relaxation rate has previously been observed for cyanine dye J-aggregates on a surface, with the radiative component of the decay rate $\left(k_{R}\right)$ predicted to increase linearly with the number of coherently 
coupled monomers. ${ }^{37-40}$ Changes in the internal conversion rate $\left(k_{i c}\right)$ with Carbz-PAHTDTT dye aggregation may also play a role in modifying the excited state relaxation rate.

As internal conversion and fluorescence are the primary excited state relaxation mechanisms for the dye monomer, with alumina behaving as an inert support substrate, the measured lifetime for the Carbz-Al-25:1 NPs should be close to the intrinsic lifetime of the Carbz-PAHTDTT molecule. Indeed, our gas-phase lifetime for the surface-bound CarbzPAHTDTT monomer is comparable to the lifetimes of similar organic dye sensitizers in solution. ${ }^{41,42}$ However, it is important to note that the lifetime of an excited state possessing charge-transfer character is sensitive to the solvent medium. ${ }^{42}$ For the Carbz-NPs, the dye's environment is probably somewhere between that of a non-polar solution, due to the surrounding CDCA molecules, and a vacuum.

The time-resolved fluorescence decay curves recorded for the gas-phase Carbz-Ti-0:1 and Carbz-Ti-25:1 samples are shown in Fig. 6, together with the decay curves for the corresponding Carbz-Al samples. It is immediately noticeable that the Carbz-Ti decays are much faster than the Carbz-Al decays. This is attributed to the excited dye injecting an electron into the titania conduction band, a mechanism that is absent for alumina.

For the Carbz-Ti decays, the fits to the stretched exponential function were poor, with quite different parameters giving similar $\chi^{2}$ values. To overcome this problem and estimate lifetimes for the Carbz-Ti samples, the $\beta$ value was fixed to that found for the analogous Carbz-Al sample, reducing the number of variable parameters from three $\left(I_{0}, \tau, \beta\right)$ to two $\left(I_{0}\right.$, $\tau)$. This procedure assumes that the kinetic distribution caused by surface inhomogeneities, different dye binding modes, and dye aggregates is similar for alumina and titania (for identical CDCA:dye ratios). Fits to the Carbz-Ti-0:1 and Carbz-Ti25:1 decay curves are shown in Fig. 6, with the fitted and derived parameters for all the Carbz-Ti samples given in Table 1. Overall, the Carbz-Ti fits overestimate the decay data at short times and underestimate the decay data at long times. The estimated average lifetimes are $0.04 \mathrm{~ns}$ for Carbz-Ti-0:1 and $0.06 \mathrm{~ns}$ for the three Carbz-Ti samples containing CDCA (i.e., Carbz-Ti-1:1, Carbz-Ti-5:1, Carbz-Ti-25:1). Deriving more accurate parameters for the ultrafast decay should be possible by using shorter excitation laser pulses (e.g., fs).

Assuming that the shorter lifetimes for Carbz-Ti, compared to Carbz-Al, are entirely due to electron injection, with rates for the remaining excited state decay processes (i.e., $k_{R}, k_{i c}$ ) and level of dye aggregation being the same for alumina and titania NPs, the electron injection lifetimes can be estimated:

$$
<\tau_{i n j}>\approx\left(k_{T i}-k_{A l}\right)^{-1}=\left(\frac{1}{<\tau_{T i}>}-\frac{1}{<\tau_{A l}>}\right)^{-1}
$$

Table 1 Gas-phase Carbz-Al and Carbz-Ti parameters derived from the emission spectra and fits of the time-resolved fluorescence decay curves to Eqns. 2-6.

\begin{tabular}{lcccccc}
\hline & $\begin{array}{c}\lambda_{\max } \\
(\mathrm{nm})\end{array}$ & $\begin{array}{c}\tau \\
(\mathrm{ns})\end{array}$ & $\beta$ & $\begin{array}{c}<\tau> \\
(\mathrm{ns})\end{array}$ & $\begin{array}{c}<\tau_{\text {inj }}> \\
(\mathrm{ns})\end{array}$ & $\Phi_{\text {inj }}$ \\
\hline Carbz-Al-0:1 & 680 & 0.04 & 0.39 & 0.14 & & \\
Carbz-Al-1:1 & 660 & 0.14 & 0.46 & 0.33 & & \\
Carbz-Al-5:1 & 645 & 0.19 & 0.48 & 0.41 & & \\
Carbz-Al-25:1 & 645 & 0.22 & 0.48 & 0.45 & & \\
Carbz-Ti-0:1 & 705 & 0.01 & $0.39^{a}$ & 0.04 & 0.06 & 0.71 \\
Carbz-Ti-1:1 & 690 & 0.03 & $0.46^{a}$ & 0.06 & 0.07 & 0.82 \\
Carbz-Ti-5:1 & 685 & 0.03 & $0.48^{a}$ & 0.06 & 0.07 & 0.85 \\
Carbz-Ti-25:1 & 685 & 0.03 & $0.48^{a}$ & 0.06 & 0.07 & 0.87 \\
\hline${ }^{a}$ value fixed
\end{tabular}

${ }^{a}$ value fixed

The electron injection lifetimes are very similar for all the Carbz-Ti samples ( $\sim 0.07 \mathrm{~ns})$, suggesting that the injection rate for dye monomers and aggregates is comparable. Although the electron injection lifetimes are short, which is ideal to inhibit reductive quenching of the dye's excited state by the oxidised redox partner within a DSSC, for the dye to provide high DSSC conversion efficiencies it is also crucial that the injection efficiency is maximized. The electron injection quantum yield $\left(\Phi_{i n j}\right)$ can be estimated from the derived Carbz-Al and Carbz-Ti lifetimes:

$$
\Phi_{i n j}=\frac{k_{i n j}}{k_{R}+k_{i c}+k_{i n j}} \approx 1-\frac{<\tau_{T i}>}{<\tau_{A l}>}
$$

The estimated injection yields for Carbz-Ti-0:1, Carbz-Ti1:1, Carbz-Ti-5:1, and Carbz-Ti-25:1 are 0.71, 0.82, 0.85, and 0.87 , respectively. Ultimately, the lower injection efficiency for the Carbz-PAHTDTT dye sensitizer without the CDCA coabsorber is a consequence of dye aggregation, which as shown for the Carbz-Al NPs, increases $k_{R}+k_{i c}$, thereby decreasing the injection probability.

Our gas-phase data provide zero-order benchmarks for aggregation, relaxation, and injection phenomena for NPs sensitized with Carbz-PAHTDTT. Currently, there are no corresponding photodynamical measurements for CarbzPAHTDTT-sensitized NPs or films surrounded by either solution or gas at atmospheric pressure. Significantly, one can expect that the CDCA:dye ratio at which dye aggregation is eliminated will be the same for gas-phase and solution-phase. However, presence of a solvent or gas may affect $k_{R}, k_{i c}$, and $k_{\text {inj }}$, and also introduce new decay processes (e.g., quenching of the $S_{1}$ state by molecular oxygen), thereby altering the electron injection efficiency. 


\subsection{Properties of Carbz-PAHTDTT-based DSSC systems}

For the dye monomer (i.e., Carbz-Ti-25:1), the gas-phase electron injection rate is estimated to be $1.4 \times 10^{10} \mathrm{~s}^{-1}$, which is commensurate with rates determined for other organic dye sensitizers. ${ }^{7}$ For example, the electron injection rate of the D149 indoline-based dye $\left(\mathrm{S}_{1} \leftarrow \mathrm{S}_{0}\right.$ maximum $\left.\sim 530 \mathrm{~nm}\right)$ has been measured in separate studies to be $3.8 \times 10^{11}$ and $3.0 \times 10^{9} \mathrm{~s}^{-1} .43,44$

The estimated Carbz-PAHTDTT injection rate complements rates derived for other operational processes within Carbz-PAHTDTT-based DSSC systems. Specifically, Daeneke et al. examined Carbz-PAHTDTT-sensitized titania electrodes in conjunction with several ferrocene/ferrocenium redox couples to determine the rates for dye cation regeneration by the redox couple and recombination of injected electrons with either the dye cation or the oxidized redox partner. ${ }^{45}$ Despite our finding that the electron injection quantum yield plateaus for CDCA:dye ratios exceeding 25:1, the most efficient Carbz-PAHTDTT-based DSSC was obtained with a far higher ratio of 1:100. ${ }^{4}$ This suggests that although such a high concentration of CDCA limits the available number of light-harvesting centers and is well in excess of that required to prevent dye aggregation, substantial benefits are attained elsewhere, such as an upward shift in the titania conduction band or protection of the surface against parasitic recombination mechanisms.

\section{Conclusions}

In conclusion, we have demonstrated that the photophysical properties of dye-sensitized NPs can be explored in the gas phase by introducing them into an ion trap via LIAD and characterizing them using emission spectroscopy and timeresolved fluorescence measurements.

This experimental setup is used to study the CarbzPAHTDTT organic dye sensitizer when attached on the surface of metal-oxide NPs, in the presence of varying concentrations of CDCA coabsorber. It is found that a CDCA:dye ratio of 25:1 is sufficient to prevent dye aggregate formation, leaving only dye monomers on the surface. For alumina NPs, the dye's emission band is blue-shifted and the excited state lifetime is increased upon coabsorption with CDCA. An emission maximum at $645 \mathrm{~nm}$ and an excited state lifetime of $0.45 \mathrm{~ns}$ is found for the dye monomer on alumina. For titania NPs, faster excited state decays result from rapid electron injection, with the lifetime for this process estimated to be $0.07 \mathrm{~ns}$. The electron injection quantum yield is lower for the dye-sensitized titania NPs without CDCA ( 0.71) compared to those with CDCA ( 0.87), due to the dye aggregates present in the former elevating the rate of the competing excited state relaxation process.
Overall, this study provides a systematic picture of the Carbz-PAHTDTT dye sensitizer's intrinsic light-harvesting behavior within a DSSC and supplies benchmark parameters for computational simulations, which are more manageable for in vacuo systems.

\section{Acknowledgments}

This research was supported under the Australian Research Council's Discovery Project funding scheme (Project Number DP120100100). VD acknowledges an Australian Renewable Energy Agency Postdoctoral Fellowship and support from the University of Melbourne's Early Career Researcher Grant Scheme. The picosecond laser was kindly provided by K.P. Ghiggino and T.A. Smith. VD would also like to thank S. Mathew (École Polytechnique Fédérale de Lausanne) for helpful discussions regarding the Carbz-NP preparation.

\section{References}

1 B. O'Regan and M. Grätzel, Nature, 1991, 353, 737-740.

2 A. Hagfeldt, G. Boschloo, L. Sun, L. Kloo and H. Pettersson, Chem. Rev., 2010, 110, 6595-6663.

3 A. Mishra, M. K. R. Fischer and P. Bäuerle, Angew. Chem. Int. Ed., 2009, 48, 2474-2499.

4 T. Daeneke, T.-H. Kwon, A. B. Holmes, N. W. Duffy, U. Bach and L. Spiccia, Nat. Chem., 2011, 3, 211-215.

5 A. Yella, H.-W. Lee, H. N. Tsao, C. Yi, A. K. Chandiran, M. K. Nazeeruddin, E. W.-G. Diau, C.-Y. Yeh, S. M. Zakeeruddin and M. Grätzel, Science, 2011, 334, 629-634.

6 C. Kelly and G. Meyer, Cord. Chem. Rev., 2001, 211, 295-315.

7 A. Listorti, B. O'Regan and J. R. Durrant, Chem. Mat., 2011, 23, 33813399.

8 H.-P. Lu, C.-Y. Tsai, W.-N. Yen, C.-P. Hsieh, C.-W. Lee, C.-Y. Yeh and E. W.-G. Diau, J. Phys. Chem. C, 2009, 113, 20990-20997.

9 A. Listorti, I. Lopez-Duarte, M. Victoria Martinez-Diaz, T. Torres, T. DosSantos, P. R. F. Barnes and J. R. Durrant, Energy Environ. Sci., 2010, 3, 1573-1579.

10 M. Dori, K. Seintis, E. Stathatos, G. Tsigaridas, T. Y. Lin, J. T. Lin, M. Fakis, V. Giannetas and P. Persephonis, Chem. Phys. Lett., 2013, 563, 63-69.

11 J. H. Yum, S. J. Moon, R. Humphry-Baker, P. Walter, T. Geiger, F. Nuesch, M. Grätzel and M. d. K. Nazeeruddin, Nanotechnology, 2008, 19, 424005.

12 G. de Miguel, M. Marchena, M. Ziolek, S. S. Pandey, S. Hayase and A. Douhal, J. Phys. Chem. C, 2012, 116, 12137-12148.

13 J.-H. Yum, S.-R. Jang, R. Humphry-Baker, M. Grätzel, J.-J. Cid, T. Torres and M. D. K. Nazeeruddin, Langmuir, 2008, 24, 5636-5640.

14 Z.-S. Wang, Y. Cui, Y. Dan-oh, C. Kasada, A. Shinpo and K. Hara, J. Phys. Chem. C, 2007, 111, 7224-7230.

15 K. Vinodgopal, X. Hua, R. Dahlgren, A. Lappin, L. Patterson and P. Kamat, J. Phys. Chem., 1995, 99, 10883-10889.

16 T. Hannappel, B. Burfeindt, W. Storck and F. Willig, J. Phys. Chem. B, 1997, 101, 6799-6802.

17 J. Friedrich, J. Fu, C. Hendrickson, A. Marshall and Y. Wang, Rev. Sci. Instrum., 2004, 75, 4511-4515.

18 A. T. Iavarone, D. Duft and J. H. Parks, J. Phys. Chem. A, 2006, 110, 12714-12727. 
19 K. Chingin, H. Chen, G. Gamez and R. Zenobi, J. Am. Soc. Mass Spectrom., 2009, 20, 1731-1738.

20 S. K. Sagoo and R. A. Jockusch, J. Photochem. Photobiol. A Chem., 2011, 220, 173-178.

21 J. P. Mondia, R. Sharma, J. Schäfer, W. Smith, Y. P. Zhao, Z. H. Lu and L. J. Wang, Appl. Phys. Lett., 2008, 93, 121102.

22 A. J. Trevitt, P. J. Wearne, E. J. Bieske and M. D. Schuder, Opt. Lett., 2006, 31, 2211-2213.

23 A. J. Trevitt, P. J. Wearne and E. J. Bieske, Int. J. Mass Spectrom., 2007, 262, 241-246.

24 A. J. Trevitt, P. J. Wearne and E. J. Bieske, J. Aerosol Sci., 2009, 40, 431-438.

25 A. Hagfeldt and M. Grätzel, Chem. Rev., 1995, 95, 49-68.

26 M. Xu, M. Zhang, M. Pastore, R. Li, F. De Angelis and P. Wang, Chem. Sci., 2012, 3, 976-983.

27 W. Peng, Y. Yang, M. Kang, Y. Tzeng, Z. Nie, H. Chang, W. Chang and C. Chen, Angew. Chem. Int. Ed., 2006, 45, 1423-1426.

28 S. Schlemmer, J. Illemann, S. Wellert and D. Gerlich, J. App. Phys., 2001, 90, 5410-5418.

29 M. Kasha, H. R. Rawls and M. A. El-Bayoumi, Pure Appl. Chem., 1965, 11, 371-392.

30 T.-H. Kwon, V. Armel, A. Nattestad, D. R. MacFarlane, U. Bach, S. J. Lind, K. C. Gordon, W. Tang, D. J. Jones and A. B. Holmes, J. Org. Chem., 2011, 76, 4088-4093.

31 N. Mohammadi and F. Wang, arXiv:1307.4832, 2013.

32 K. Sayama, S. Tsukagoshi, T. Mori, K. Hara, Y. Ohga, A. Shinpou, Y. Abe, S. Suga and H. Arakawa, Sol. Energ. Mat. Sol. Cells, 2003, 80, $47-71$.

33 S. E. Koops, P. R. F. Barnes, B. C. O'Regan and J. R. Durrant, J. Phys. Chem. C, 2010, 114, 8054-8061.

34 A. El-Zohry, A. Orthaber and B. Zietz, J. Phys. Chem. C, 2012, 116, 26144-26153.

35 A. R. K. Selvaraj and S. Hayase, J. Mol. Model., 2012, 18, 2099-2104.

36 H. Kusama and K. Sayama, J. Phys. Chem. C, 2012, 116, 23906-23914.

37 D. Mobius and H. Kuhn, J. App. Phys., 1988, 64, 5138-5141.

38 M. A. Ratner, Low Temp. Phys., 2003, 29, 602-605.

39 T. Tani, T. Suzumoto, K. Kemnitz and K. Yoshihara, J. Phys. Chem., 1992, 96, 2778-2783.

40 A. A. Muenter, D. V. Brumbaugh, J. Apolito, L. A. Horn, F. C. Spano and S. Mukamel, J. Phys. Chem., 1992, 96, 2783-2790.

41 K. Oum, P. W. Lohse, J. R. Klein, O. Flender, M. Scholz, A. Hagfeldt, G. Boschloo and T. Lenzer, Phys. Chem. Chem. Phys., 2013, 15, 39063916.

42 P. W. Lohse, J. Kuhnt, S. I. Druzhinin, M. Scholz, M. Ekimova, T. Oekermann, T. Lenzer and K. Oum, Phys. Chem. Chem. Phys., 2011, 13, 19632-19640.

43 M. Fakis, E. Stathatos, G. Tsigaridas, V. Giannetas and P. Persephonis, J. Phys. Chem. C, 2011, 115, 13429-13437.

44 H. J. Snaith, A. Petrozza, S. Ito, H. Miura and M. Grätzel, Adv. Funct. Mater., 2009, 19, 1810-1818.

45 T. Daeneke, A. J. Mozer, T.-H. Kwon, N. W. Duffy, A. B. Holmes, U. Bach and L. Spiccia, Energy Environ. Sci., 2012, 5, 7090-7099. 